\title{
PK+ Tree: An Improved Spatial Index Structure of PK Tree
}

\author{
Xiaolin Wang, Yingwei Luo ${ }^{*}$, Lishan Yu, and Zhuoqun Xu \\ Dept. of Computer Science and Technology, Peking University, Beijing, P.R.China, 100871 \\ lyw@pku.edu.cn
}

\begin{abstract}
Spatial index is very important in GIS. PK tree and Hilbert R tree are two well-known spatial index structures. Comparison operations are very little in PK tree, while disk I/O operations are quite little in Hilbert R tree. PK+ Tree is an improved spatial index structure from PK tree. In PK+ tree, Comparison operations are less than in Hilbert $\mathrm{R}$ tree, while disk I/O operations are almost the same as in Hilbert R tree.
\end{abstract}

\section{Introduction}

With the development of information technology about data mining and multimedia, massive data processing gets more and more important in spatial processing. Spatial indexing is a key issue for massive spatial data processing.

Many kinds of spatial index structures exist nowadays. They can be divided into two main categories: partition tree and $\mathrm{R}$ tree. These two categories of spatial index structure have their own weakness and strongpoint. PK tree and Hilbert R tree are two efficient spatial index structures of each category.

In this paper, a new spatial index structure, $\mathrm{PK}+$ tree, which are from PK tree, is introduced in order to improve storage usage, so that it may be more efficient for high dimensional data. In the next section, the problems of PK tree are analyzed. In the third section, PK+ tree is discussed in details. In the fourth section, the efficiency of storage and query of $\mathrm{PK}+$ tree are compared with of PK tree and Hilbert R tree.

\section{PK Tree and Its Weakness}

PK tree is an efficient spatial index structure of partition tree category. In PK tree, an initial space cell $C_{0}$ are required to enclosing all the data objects to be indexed. $C_{0}$ can be split into $R=\left(r_{x}, r_{y}\right)$ sub cells $C_{l}$, and so on, so that the width and height of each $C_{i}$ is $1 / r_{x}$ and $1 / r_{y}$ of $C_{i-1}$. Only those $\mathrm{K}$-instantialable cells have their corresponding nodes in PK tree.

A key issue of PK tree is that it's poor efficiency of storage. The average node usage (ANU) is the percentage of valid entries to the capacity of entries in the tree. For data points in two dimensional space, each node has the capacity to hold $R(K-1)$ en-

*Corresponding author: LUO Yingwei, lyw@pku.edu.cn. 
tries, but the ANU is only $40 \%$. For the same data set, the ANU of a Hilbert R tree may reach to $84 \%$ or $87 \%$. When processing data object other than point, for example curves or surfaces, the ANU of a PK tree can reach only $12 \%$, while Hilbert R tree may also reach around $85 \%$. The poor efficiency of storage leads to more disk I/O operations while access the index tree. For data point, the ANU of PK tree is half of the ANU of Hilbert R tree, but PK tree yells more efficient query performance than Hilbert R tree. But for curves or surfaces, the query performance of PK tree is much poorer than Hilbert $\mathrm{R}$ tree. The main reason is that the ANU of PK tree is far more lees than that of Hilbert R tree. With such poor ANU, PK tree are limited to be used for high dimensional data object. To solve this problem, $\mathrm{PK}+$ tree is introduced in this paper aiming at improving the ANU.

\section{PK+ Tree}

The ANU of PK tree drops with large $R=\left(r_{x}, r_{y}\right)$. If $R$ can be limited to the smallest value, then the ANU might be greatly increased. In $\mathrm{PK}+$ tree, $R$ is restricted to be no more than 3. To partition a given space cell $C$ in two-dimensional space, firstly $C$ is split into three cells at $\mathrm{x}$ dimension, then at $\mathrm{y}$ dimension, and then at $\mathrm{x}$ dimension, and so on. The three cells are not symmetrical. Two cells of them are the two half of $C$, while the third cell is $C$ itself! But the third cell is not the same as $C$, since is hold all data objects sits in $C$ but not in the other two half cells. The third cell is called middle cell, and the two half-cells are called left cell and right cell (or bottom cell and top cell) correspondingly, as shown in figure 1 .

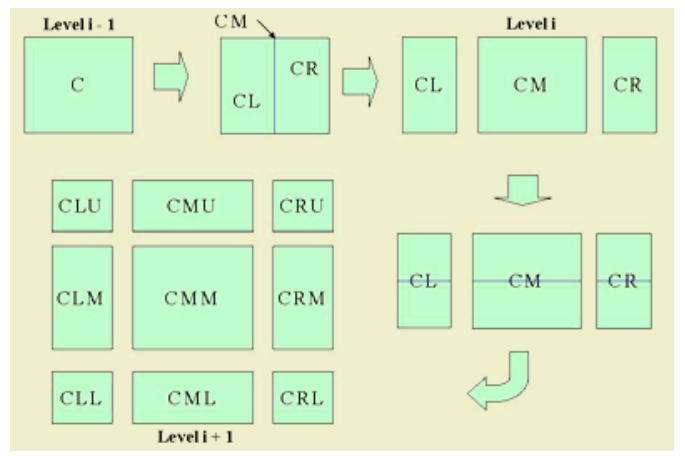

Fig. 1. PK+ Tree Cell Splitting

$\mathrm{PK}+$ tree also requires that any node in the tree is K-intantialable. Insertion or deletion is implemented with two steps. First, search from the root to find the proper node to be modified, and modify it; then, trace back to the root and checking each node on the path if it's K-intantialable, if not, some promotion, division or deletion operations will be done to keep all nodes $\mathrm{K}$-intantialable. The process of K-intantialable checking in $\mathrm{PK}+$ tree is the same as in PK tree, except that they apply different kinds of splitting rules. 
$\mathrm{PK}+$ tree split the space cell at each dimension in turn, which makes a lower degree of regularity of partition than PK tree, but the ANU is greatly increased. In PK+ tree, the extent of a middle node overlaps with its sibling left (right) node. The overlapping of nodes might reduce some query's performance.

\section{Performance Comparison}

In the section, a series of experiments are done to examine the query performance of $\mathrm{PK}+$ tree. Two dimensional data objects in two-dimensional space are being used in our experiment. A cluster data set from SEQUOIA 2000 Benchmark and a random data set are used. In these experiments, we compare $\mathrm{PK}+$ tree with corresponding $\mathrm{PK}$ tree and 3-2 Hilbert R tree.

We count disk I/O operations and compare operations instead of measure the time cost in queries as the measurement of performance, thus we avoid the effects of implementation issues to our experiments.

Since in PK tree and PK+ tree, theoretically, the ANU will never be large than $50 \%$. To make the storage efficiently, two kinds of methods might be applied. One method is to store each node in one or more disk pages according to the amount of valid entries in each node (SNMP, Single-node-multi-pages). The other method is to store several nodes in the same disk page if the total valid entries can be hold in one disk page (MNSP, Multi-nodes-single-page).

\subsection{Storage Usage}

For a given data set, the storage usage is the total valid entries vs. the total entries capacity of the index structure. ANU is used to describe the storage usage.

Table 1 shows the comparison of storage usage of PK tree, PK+ tree and Hilbert R tree. SNMP is compared with MNSP to show the effect of MNSP. Since Hilbert R tree is storage optimized, no more optimized method is considered in the comparison.

Table 1. ANU of $P K+$ tree, $P K$ tree and Hilbert $R$ tree

\begin{tabular}{|c|c|c|c|c|}
\hline \multirow{2}{*}{} & \multicolumn{2}{|c|}{ SNMP } & \multicolumn{2}{c|}{ MNSP } \\
\cline { 2 - 5 } & Cluster data & Random data & Cluster data & Random data \\
\hline PK+ tree & $46.37 \%$ & $50.83 \%$ & $67.62 \%$ & $66.78 \%$ \\
\hline PK tree & $12.95 \%$ & $12.69 \%$ & $25.49 \%$ & $38.15 \%$ \\
\hline Hilbert R tree & $88.17 \%$ & $85.52 \%$ & $\square$ & $\square$ \\
\hline
\end{tabular}

Table 1 shows that, MNSP outperforms SNMP, but does not make the ANU of $\mathrm{PK}+$ tree reach the ANU of Hilbert R tree.

\subsection{Query Performance}

Our experiments show that, for both cluster data set and random data set, $\mathrm{PK}+$ tree greatly reduces disk I/O operations per query than PK tree, but has almost the same 
disk I/O operations per query as Hilbert R tree. Since the ANU of PK+ tree is approximately $75 \%$ of Hilbert $\mathrm{R}$ tree, it is obvious that $\mathrm{PK}+$ tree requires much less compare operations per query than that of Hilbert $\mathrm{R}$ tree. In more details, for small query regions, $\mathrm{PK}+$ tree yells less disk $\mathrm{I} / \mathrm{O}$ operations than Hilbert R tree; for query of inside, $\mathrm{PK}+$ tree yells much less disk I/O operations than Hilbert R tree.

\section{Conclusion}

In this paper, an improved spatial index structure based on PK tree - PK+ tree is introduced. PK+ tree outperforms PK tree when used for high dimensional object indexing. $\mathrm{PK}+$ tree has a higher ANU and hence requires less disk I/O operations in queries. Though disk I/O operations in queries of $\mathrm{PK}+$ tree is almost the same as that of Hilbert R tree, but Hilbert R tree yells a higher ANU, compare operations in queries is more than that of $\mathrm{PK}+$ tree. $\mathrm{PK}+$ tree is perfect for application that cares both disk $\mathrm{I} / \mathrm{O}$ performance and CPU performance.

\section{Acknowledgement}

This work is supported by the National Research Foundation for the Doctoral Program of Higher Education of China under Grant No. 20020001015; the National Grand Fundamental Research 973 Program of China under Grant No.2002CB312000; the National Science Foundation of China under Grant No.60203002; the National High Technology Development 863 Program under Grant No. 2002AA135330 and No. 2002AA134030; the Beijing Science Foundation under Grant No.4012007.

\section{References}

1. Wei Wang, Jiong Yang and Richard Muntz: PK-tree: A Dynamic Spatial Index Structure for Large Data Sets, Kluwer Academic Publishers (1997)

2. Wei Wang, Jiong Yang and Richard Muntz: Pk-Tree: A Spatial Index Structure For High Dimensional Point Data, Kluwer Academic Publishers (2000).

3. Ibrahim Kamel and Christos Faloutsos: Hilbert R-tree: An improved R-tree Using Fractals, Morgan Kaufmann Publishers Inc (1994).

4. Bernhard Seeger and Hans-Peter Kriegel: Techniques for Design and Implementation of Efficient Spatial Access Methods, Morgan Kaufmann Publishers Inc (1988).

5. Andreas Henrich, Hanas-Werner Six and Peter Widmayer: The LSD Tree: Spatial Access to Multidimensional Point and Non-point Objects, Morgan Kaufmann Publishers Inc (1989). 